\title{
Investigation of airborne fungi at different altitudes in Shenzhen University
}

\author{
Li Li, Chao Lei, Zhi-Gang Liu*
}

College of Life Science, Shenzhen University, Shenzhen, China; ${ }^{*}$ Corresponding Author: LZG@,szu.edu.cn

Received 15 December 2009; revised 24 February 2010; accepted 18 March 2010.

\begin{abstract}
Aim: To investigate the richness of species or genera of airborne fungi, the amount of airborne fungi, and its seasonal variation at different altitudes in Shenzhen University. The effect of meteorological factors on airborne fungi was also analyzed. Methods: Slide-exposure method and open-plate method were used. Results: There were 27 genera or species of fungus spores identified. Among the identified fungal genus, Cladosporium, Ustilago, Alternaria, Helminthsporium and Uredinales were more prevalent. There were $\mathbf{1 8}$ genera of fungi colonies identified. Among which Penicillium, non-sporulating fungi, Aspergillus, Saccharomyces and Cladosporium were more common. The airborne fungal spores were present in the atmosphere of Shenzhen University all year round. The peaks of airborne spores appeared during April and October, while the lowest numbers were observed during January, July and December from March 2005-Febrary 2006. The highest volumes of fungi colonies were observed during April, October and September, while the lowest numbers were detected during in January, July and December or May from March 2005-Febrary 2006. The meteorological factors had no relationship between the total monthly spore count at $\mathbf{1 0}$ and $\mathbf{3 0}$ meter height. At $\mathbf{7 0}$ meter, the total spores count was negatively correlated with solar radiation. Conclusions: Most of the fungi spores decreased along with the increase of altitudes.
\end{abstract}

Keywords: Airborne Fungi; Open-Plate Method; Slide-Exposure Method

\section{INTRODUCTION}

Airborne fungi are one of the common allergens that induce respiratory hypersensitivity reaction [1-3]. The major allergic symptoms include asthma, rhinitis, bronchopulmonary mycoses and hypersensitive pneumonitis [4]. Airborne fungi also act as an indicator for the atmospheric bio-pollution. The presence of fungal propagules, volatiles and mycotoxins in the air can pose a health hazard in all segments of the population [5]. Fungi variety and concentration depends on various factors, including topography, time of day, meteorological parameters, seasonal climatic variation and type of vegetation [6-7]. Extensive investigations of airborne fungi had been done in many parts of the world [8-15]. In China, such studies had been done in different provinces [16-25].

But to our knowledge there are no published data on the airborne fungi at different altitudes in one place. Shenzhen city is located at $22^{\circ} 27^{\prime}-22^{\circ} 52^{\prime} \mathrm{N}$ and $113^{\circ} 46^{\prime}-$ $114^{\circ} 37^{\prime}$ E. The weather in Shenzhen is associated with high temperature and humidity throughout the year, which suits for the reproduction of airborne fungi. As a well developed city, Shenzen has a large number of tall buildings and mansions providing both working and living spaces. The aim of this work was to determine the concentration of airborne fungi present at different altitudes and the effect of seasonal variations, which will provide useful information on the air quality of residential areas.

\section{METHODS}

\subsection{Slide-Exposure Method}

The slide-exposure method based on the protocol adapted from Ye (1992) was used to determine the fungal spores [26]. Briefly, the slides with vaseline for spore sampling were set on the second floor (10 meter above ground level), the seventh floor (30 meter above ground level) and the sixtieth floor (70 meter above ground level) of the technological building in Shenzhen University $\left(22^{\circ} 54^{\prime} 37^{\prime \prime} \mathrm{N}\right.$ and $\left.113^{\circ} 93^{\prime} 77^{\prime \prime} \mathrm{E}\right)$, respectively. Two slides were collected daily from March, 2005 to February, 2006. The slides were stained by basic fuchsin solu- 
tion. Fungal spores were enumerated and identified by using a light microscope (OLYMPUS BH-2, Japan). The average spore counts of the two samples in each altitude were taken.

\subsection{Open-Plate Method}

The open-plate method was adapted based on Ye (1992) to determine the fungal colonies [26]. Briefly, the plates with Peptone Dextrose Agar, Potato Dextrose Agar and Czapek Dox Agar respectively were set on the same places as described above once during each month (total 12 times in one year). Three plates were opened for a period of $5 \mathrm{~min}$ at various altitudes, and then were incubated at $28^{\circ} \mathrm{C} \pm 1{ }^{\circ} \mathrm{C}$ for up to 5 days. The colonies were stained by lactic acid methylene blue solution and identified by colony and microscopic morphology. Colony counts were then converted to the colony forming units $/ \mathrm{m}^{3}, \mathrm{CFU} / \mathrm{m}^{3}=5000 \mathrm{~N} / \mathrm{A} \mathrm{t}(\mathrm{N}$ : colony count; A: square of plate; $\mathrm{t}$ : exposure time) [27]. The total colony forming units of three plates at each altitude were added to obtain the total colony forming units at each altitude.

\subsection{Statistical Analysis}

The relationship between the monthly total spores count at various altitudes, the most common fungi and the monthly meteorological factors (average temperature: $\mathrm{X} 1$; average relative humidity: $\mathrm{X} 2$; average atmospheric pressure: X3; average wind speed: X4; rainfall: X5 and solar radiation: X6) were established respectively by means of stepwise multiple regression method (The data were analyzed using SPSS Version 12.0). The value of $\mathrm{p}<0.05$ was considered statistically significant. The meteorlogical data were obtained from Shenzhen meteorlogical administration (Table 1).

\section{RESULTS}

\subsection{Airborne Fungal Spore Count at Three Different Altitudes}

During the entire year, there were 2190 slides collected at three different altitudes. The total fungal spore counts at 10, 30 and 70 meters height were 4,102, 3,540 and
2,929.5 respectively. There were 27 genera or species identified belonging to the subphylum Zygomycotina family Mucoraceae: Rhizopus (0.17\%); subphylum Ascomycotina family Sphaeriaceae: Chaetomium $(0.39 \%)$; subphylum Basidiomycotina family Ustilaginaceae: Ustilago (13.04\%), Uredinales (9.54\%); subphylum Deuteromycotina family Moniliaceae: Cladosporium (16.45\%), Aspergillus (0.59\%), Geotrichum (0.52\%), Botrytis (0.31\%), Trichothecium (0.09\%), family Dematiaceae: Alternaria (11.41\%), Helminthosporium (11.37\%), Curvularia (8.41\%), Stachybotrys (5.38\%), Stemphylium (4.58\%), Nigrospora (1.93\%), Heterosporium (1.02\%), Acrothecium sp. (0.53\%), Papularia (0.31\%), Clavispora (0.22\%), Cercospora sp. (0.09\%), Wardomyces $(0.05 \%)$, family Tuborculariaceae: Fusarium (0.85\%), Epicoccum (0.48\%), family Sphaeropsidaceae: Hendersonia $s p$. (4.18\%), Ascochyta sp. (4.04\%), Diplodia sp. (3.65\%), Sphaeropsis sp. $(0.20 \%)$ and unidentified spores $(0.20 \%)$ (Tables 2-4).

At three different altitudes, the majority of spores were Cladosporium, Ustilago, Alternaria, Helminthosporium and Uredinales.

\subsection{Fungal Colony Forming Units at Three Different Altitudes}

During the entire year, the total of 53,473, 50,962 and 49,543 colony forming units at 10,30 and 70 meters, respectively were collected, enumerated and then characterized into 18 genera or species. The common fungi belonged to the subphylum Zygomycotina family $\mathrm{Mu}$ coraceae: Mucor (0.31\%), Rhizopus (0.31\%); subphylum Ascomycotina family Sphaeriaceae: Chaetomium (0.51\%), family Saccharomyetaceae: Saccharomyces (12.67\%); subphylum Deuteromycotina family Moniliaceae: Penicillium (24.11\%), Aspergillus (15.63\%), Cladosporium (11.85\%), Trichothecium (0.71\%), Trichoderma $(0.72 \%)$, Botrytis $(0.51 \%)$, Geotrichum $(0.31 \%)$, family Dematiaceae: Alternaria (5.11\%), Curvularia (5.00\%), Helminthosporium (1.22\%), Nigrospora (0.51\%), Stachybotrys $(0.51 \%)$, family Tuborculariaceae: Fusarium (0.71\%), family Sphaeropsidaceae: Phoma sp. (0.61\%), non-sporulating fungi $(18.28 \%)$ and unidentified colony $(0.41 \%)$ (Tables 5-7).

Table 1. Averages of meteorological measurements: temperature (T), relative humidity $(\mathrm{RH})$, atmospheric pressure $(\mathrm{P})$, wind speed (WS), rainfall (R), and solar radiation (SR) in Shenzhen from March 2005-Febrary 2006.

\begin{tabular}{lcccccccccccc}
\hline & Mar & Apr & May & Jun & Jul & Aug & Sep & Oct & Nov & Dec & Jan & Feb \\
\hline T ( $\left.{ }^{\circ} \mathbf{C}\right)$ & 17.2 & 23.1 & 27.2 & 27.8 & 29.4 & 28.5 & 28.8 & 26.2 & 22.9 & 16.7 & 16.2 & 17.8 \\
RH (\%) & 71 & 74 & 76 & 78 & 71 & 79 & 72 & 64 & 67 & 50 & 69 & 72 \\
P (hPa) & 1016.6 & 1011.9 & 1005.6 & 1002.4 & 1004.3 & 1002.8 & 1007.2 & 1012.8 & 1014.4 & 1019.2 & 1016.4 & 1017.6 \\
WS (m/sec) & 2.1 & 1.8 & 1.8 & 1.7 & 1.7 & 1.9 & 2.2 & 2.4 & 2.2 & 2.7 & 2.6 & 2.3 \\
R (mm) & 48.3 & 42.9 & 379.2 & 469.9 & 326.6 & 587.3 & 231.8 & 21.3 & 14.0 & 9.0 & 20.6 & 48.0 \\
SR (h) & 759 & 881 & 1194 & 756 & 2264 & 1639 & 1495 & 1946 & 1798 & 1549 & 1202 & 912 \\
\hline
\end{tabular}


Table 2. The identified airborne fungi genera or species and slide fungal spores count at 10 meter during 12 months.

\begin{tabular}{|c|c|c|c|c|c|c|c|c|c|c|c|c|c|}
\hline or species & Jan & Feb & Mar & Apr & May & Jun & Jul & Aug & Sep & Oct & Nov & Dec & Total spores \\
\hline Rhizopus & 0 & 0 & 0 & 2 & 0 & 0 & 2.5 & 0 & 0 & 0 & 0 & 0 & 4.5 \\
\hline Chaetomium & 0 & 2 & 1 & 0 & 2.5 & 0 & 2 & 4.5 & 1.5 & 1.5 & 1 & 4 & 20 \\
\hline Ustilago & 23.5 & 67.5 & 80.5 & 37.5 & 56.5 & 22.5 & 15 & 31 & 81 & 57.5 & 23.5 & 42.5 & 538.5 \\
\hline Uredinales & 40.5 & 36 & 40 & 29 & 26.5 & 30.5 & 32 & 30.5 & 30 & 32.5 & 47.5 & 15.5 & 390.5 \\
\hline Cladosporium & 47 & 21.5 & 19 & 90.5 & 103 & 52 & 32.5 & 52.5 & 15.5 & 97.5 & 72.5 & 22 & 625.5 \\
\hline Aspergillus & 2.5 & 3 & 0 & 3 & 1 & 1 & 3 & 0 & 2 & 3.5 & 2.5 & 0 & 21.5 \\
\hline Geotrichum & 6.5 & 0 & 0 & 2 & 0 & 0 & 2.5 & 0 & 3 & 0 & 2 & 1.5 & 17.5 \\
\hline Botrytis & 0 & 2 & 1.5 & 0 & 3 & 2 & 0 & 1.5 & 3.5 & 0 & 0 & 5.5 & 19 \\
\hline Trichothecium & 0 & 1 & 0 & 0 & 0 & 0 & 0 & 0 & 0 & 0 & 0 & 3 & 4 \\
\hline Alternaria & 42 & 26.5 & 33.5 & 81 & 46.5 & 37 & 34.5 & 52 & 40 & 37 & 30 & 25.5 & 485.5 \\
\hline Helminthosporium & 24 & 40.5 & 68 & 22.5 & 42 & 48 & 24.5 & 30 & 34 & 58 & 31.5 & 34 & 457 \\
\hline Curvularia & 24 & 34 & 24.5 & 36.5 & 31.5 & 29 & 15 & 15.5 & 30.5 & 44 & 39.5 & 23 & 347 \\
\hline Stachybotrys & 18 & 20 & 13 & 33 & 15 & 14.5 & 9.5 & 20 & 20.5 & 24.5 & 18 & 23.5 & 229.5 \\
\hline Stemphylium & 15 & 15.5 & 15 & 18.5 & 16 & 14.5 & 13 & 15.5 & 23.5 & 17.5 & 21.5 & 20 & 205.5 \\
\hline Nigrospora & 6.5 & 10.5 & 15 & 6.5 & 4 & 5.5 & 10 & 5 & 3.5 & 7.5 & 6 & 6.5 & 86.5 \\
\hline Heterosporium & 5.5 & 0 & 1.5 & 6 & 2 & 2 & 1.5 & 0 & 5.5 & 5.5 & 0 & 0 & 29.5 \\
\hline Acrothecium sp. & 2 & 0 & 2 & 3 & 3 & 3 & 2.5 & 3 & 4.5 & 0.5 & 1 & 4.5 & 29 \\
\hline Papularia & 0 & 0 & 0 & 0 & 0 & 1.5 & 0 & 0 & 3.5 & 5 & 4.5 & 0 & 14.5 \\
\hline Clavispora & 1 & 0.5 & 1 & 0 & 0.5 & 0 & 0 & 1 & 0.5 & 0 & 0 & 0 & 4.5 \\
\hline Cercospora sp. & 0 & 0 & 2 & 0 & 0 & 0 & 1 & 0 & 0 & 0.5 & 0 & 1 & 4.5 \\
\hline Wardomyces & 0 & 0 & 0 & 0 & 0 & 0 & 1 & 0 & 0 & 0 & 0 & 0.5 & 1.5 \\
\hline Fusarium & 4 & 1.5 & 1 & 2.5 & 1.5 & 2 & 0 & 0 & 2.5 & 2.5 & 2.5 & 0 & 20 \\
\hline Epicoccum & 2 & 2.5 & 1 & 2.5 & 0 & 1.5 & 0 & 2 & 2.5 & 5.5 & 0 & 2.5 & 22 \\
\hline Hendersonia sp. & 15 & 16 & 27 & 18.5 & 15 & 9.5 & 13 & 10.5 & 19.5 & 16.5 & 9 & 24.5 & 194 \\
\hline Ascochyta sp. & 9 & 7.5 & 11 & 16.5 & 7.5 & 10.5 & 15 & 15 & 17 & 15.5 & 19.5 & 23 & 167 \\
\hline Diplodia sp. & 20 & 7.5 & 16 & 16.5 & 11.5 & 8 & 11 & 8 & 15.5 & 12 & 12 & 7 & 145 \\
\hline Sphaeropsis sp. & 2.5 & 0 & 5 & 0 & 0 & 0 & 0 & 1 & 0 & 0 & 0 & 1.5 & 10 \\
\hline Unidentified spores & 2.5 & 0.5 & 1 & 0 & 0 & 0 & 1 & 0.5 & 0 & 1 & 0.5 & 1.5 & 8.5 \\
\hline Total spores & 313 & 316 & 379.5 & 427.5 & 388.5 & 294.5 & 242 & 299 & 359.5 & 445.5 & 344.5 & 292.5 & 4102 \\
\hline
\end{tabular}

Table 3. The identified airborne fungi genera or species and slide fungal spores count at 30 meter during 12 months.

\begin{tabular}{|c|c|c|c|c|c|c|c|c|c|c|c|c|c|}
\hline or species & Jan & Feb & Mar & Apr & May & Jun & Jul & Aug & Sep & Oct & Nov & Dec & Total spores \\
\hline Rhizopus & 0 & 0 & 1.5 & 2.5 & 0 & 0 & 1 & 0 & 0 & 0 & 0 & 4 & 9 \\
\hline Chaetomium & 0.5 & 1.5 & 2.5 & 0 & 1.5 & 0 & 1.5 & 2.5 & 0.5 & 1.5 & 0.5 & 1.5 & 14 \\
\hline Ustilago & 26.5 & 33.5 & 59 & 47 & 26 & 22.5 & 16.5 & 25.5 & 68 & 62.5 & 24.5 & 32.5 & 444 \\
\hline Uredinales & 34.5 & 30.5 & 31.5 & 32.5 & 15.5 & 24 & 26 & 23 & 23 & 40.5 & 31 & 22.5 & 335 \\
\hline Cladosporium & 21.5 & 28.5 & 29.5 & 86 & 56.5 & 46 & 32.5 & 43 & 22.5 & 85.5 & 54 & 38.5 & 544 \\
\hline Aspergillus & 1.5 & 2 & 2 & 2.5 & 2 & 3 & 0 & 2 & 3 & 1.5 & 0 & 2 & 21.5 \\
\hline Geotrichum & 2 & 0 & 1 & 3 & 2 & 0 & 1.5 & 0 & 3.5 & 0 & 1 & 1.5 & 15.5 \\
\hline Botrytis & 0 & 1.5 & 0.5 & 0 & 1.5 & 2.5 & 0 & 1 & 1 & 0 & 0 & 3 & 11 \\
\hline Trichothecium & 0 & 0.5 & 0 & 0 & 2 & 0 & 0 & 0 & 0 & 0 & 0 & 1 & 3.5 \\
\hline Alternaria & 37.5 & 23.5 & 21 & 56 & 40.5 & 28.5 & 31.5 & 42 & 23.5 & 34.5 & 24.5 & 29 & 392 \\
\hline Helminthosporium & 16 & 32 & 62 & 45.5 & 36.5 & 43 & 14 & 27 & 27.5 & 44.5 & 24 & 24.5 & 397 \\
\hline Curvularia & 20.5 & 24.5 & 32 & 36 & 33.5 & 36 & 17 & 17 & 23.5 & 38.5 & 34.5 & 16 & 329 \\
\hline Stachybotrys & 12 & 17.5 & 18 & 18 & 20.5 & 16 & 12 & 17.5 & 15.5 & 25.5 & 19 & 7.5 & 199 \\
\hline Stemphylium & 8 & 12.5 & 11 & 14 & 20.5 & 19.5 & 10 & 12 & 20.5 & 14 & 17 & 15 & 174 \\
\hline Nigrospora & 8 & 7.5 & 10 & 10 & 3.5 & 4.5 & 7 & 4 & 1.5 & 9.5 & 4 & 8.5 & 78 \\
\hline Heterosporium & 3.5 & 0.5 & 1.5 & 5.5 & 3.5 & 2.5 & 1 & 0 & 4.5 & 5.5 & 0.5 & 2 & 30.5 \\
\hline Acrothecium sp. & 1 & 0 & 0 & 1.5 & 1.5 & 1 & 1.5 & 2.5 & 3.5 & 0 & 1.5 & 2 & 16 \\
\hline Papularia & 0 & 1 & 0 & 0 & 1.5 & 2.5 & 0 & 0 & 2 & 3 & 2.5 & 0 & 12.5 \\
\hline Clavispora & 1 & 1.5 & 0.5 & 1 & 0 & 0.5 & 0 & 0 & 1 & 1.5 & 1 & 1 & 9 \\
\hline Cercospora sp. & 0 & 0 & 0.5 & 0 & 0.5 & 0 & 0 & 0 & 0 & 0 & 0 & 0 & 1 \\
\hline Wardomyces & 0 & 0 & 0 & 0 & 0.5 & 0.5 & 0 & 0 & 0 & 0 & 0 & 0 & 1 \\
\hline Fusarium & 2 & 2.5 & 2.5 & 4 & 2.5 & 4 & 1 & 0 & 4 & 4.5 & 1.5 & 0 & 28.5 \\
\hline Eрісоссит & 1 & 1 & 1 & 0 & 0 & 3.5 & 1.5 & 3.5 & 1 & 4.5 & 0 & 0 & 17 \\
\hline Hendersonia sp. & 19 & 8.5 & 22 & 15.5 & 11 & 9 & 10 & 8 & 15 & 13.5 & 6 & 14.5 & 152 \\
\hline Ascochyta sp. & 13 & 17.5 & 25.5 & 17 & 9 & 7.5 & 13 & 12 & 12 & 12 & 9.5 & 14.5 & 163 \\
\hline Diplodia sp. & 13.5 & 14 & 21.5 & 12 & 7 & 6 & 6 & 10.5 & 10.5 & 12.5 & 9.5 & 5 & 128 \\
\hline Sphaeropsis sp. & 0 & 0 & 2.5 & 0 & 2.5 & 0 & 0 & 0 & 1.5 & 0 & 0 & 2.5 & 9 \\
\hline Unidentified spores & 1 & 1 & 0.5 & 0 & 1 & 1 & 0.5 & 0 & 0 & 0 & 0.5 & 2 & 7.5 \\
\hline Total spores & 243.5 & 263 & 359.5 & 409.5 & 302.5 & 283.5 & 205 & 253 & 288.5 & 415 & 266.5 & 250.5 & 3540 \\
\hline
\end{tabular}


Table 4. The identified airborne fungi genera or species and slide fungal spores count at 70 meter during 12 months.

\begin{tabular}{|c|c|c|c|c|c|c|c|c|c|c|c|c|c|}
\hline $\begin{array}{l}\text { Genera month } \\
\text { or species }\end{array}$ & Jan & Feb & Mar & Apr & May & Jun & Jul & Aug & Sep & Oct & Nov & Dec & $\begin{array}{c}\text { Total } \\
\text { spores }\end{array}$ \\
\hline Rhizopus & 0 & 0 & 0 & 3 & 0 & 0 & 0 & 0 & 0 & 0 & 1.5 & 0 & 4.5 \\
\hline Chaetomium & 0 & 2 & 0.5 & 1 & 2 & 0 & 0 & 0.5 & 0 & 0 & 0 & 1 & 7 \\
\hline Ustilago & 18.5 & 46.5 & 30.5 & 44.5 & 23.5 & 32 & 21 & 30.5 & 74.5 & 26.5 & 31 & 16.5 & 395.5 \\
\hline Uredinales & 13 & 19 & 40.5 & 43.5 & 19.5 & 14.5 & 20.5 & 15 & 12 & 19.5 & 35.5 & 30 & 282.5 \\
\hline Cladosporium & 29.5 & 16.5 & 95.5 & 88.5 & 33 & 36 & 19.5 & 36 & 20.5 & 65 & 65 & 65 & 570 \\
\hline Aspergillus & 2.5 & 0 & 1.5 & 3.5 & 3 & 1.5 & 0 & 2.5 & 2 & 1.5 & 0 & 1 & 19 \\
\hline Geotrichum & 3.5 & 0 & 1.5 & 5 & 5 & 0 & 0.5 & 0 & 3 & 0 & 1 & 2 & 21.5 \\
\hline Botrytis & 0 & 0 & 0.5 & 0 & 0.5 & 2 & 0 & 0 & 0 & 0 & 0 & 0 & 3 \\
\hline Trichothecium & 0 & 0 & 0 & 0 & 2.5 & 0 & 0 & 0 & 0 & 0 & 0 & 0 & 2.5 \\
\hline Alternaria & 27 & 35 & 40.5 & 44.5 & 30 & 27 & 20 & 24 & 15.5 & 19.5 & 15 & 31 & 329 \\
\hline Helminthosporium & 24.5 & 38 & 39 & 55 & 29.5 & 32.5 & 18.5 & 36 & 19 & 32 & 12 & 12 & 348 \\
\hline Curvularia & 6 & 28.5 & 10 & 25 & 23 & 18.5 & 12.5 & 22 & 19.5 & 21 & 18.5 & 9 & 213.5 \\
\hline Stachybotrys & 0 & 12 & 9.5 & 13.5 & 18.5 & 15.5 & 10 & 15 & 8.5 & 16.5 & 10.5 & 10.5 & 140 \\
\hline Stemphylium & 4 & 6 & 8 & 9.5 & 12.5 & 10.5 & 6 & 7 & 12.5 & 9 & 10.5 & 9 & 104.5 \\
\hline Nigrospora & 3 & 4.5 & 9 & 3.5 & 2 & 2 & 6 & 2 & 0.5 & 1.5 & 2 & 3.5 & 39.5 \\
\hline Heterosporium & 8.5 & 1.5 & 2.5 & 2 & 6.5 & 5 & 0 & 1.5 & 7.5 & 6.5 & 2.5 & 4 & 48 \\
\hline Acrothecium sp. & 1 & 0 & 1 & 1 & 1.5 & 0 & 0.5 & 1 & 2 & 2 & 0.5 & 1 & 11.5 \\
\hline Papularia & 0 & 2 & 0 & 0 & 1 & 1.5 & 0 & 0 & 1.5 & 0 & 0 & 0 & 6 \\
\hline Clavispora & 4 & 1 & 1.5 & 0.5 & 1 & 1 & 0 & 0 & 0 & 0 & 0 & 1 & 10 \\
\hline Cercospora sp. & 0 & 0 & 3.5 & 0 & 0.5 & 0 & 0 & 0 & 0 & 0 & 0 & 0 & 4 \\
\hline Wardomyces & 0 & 0 & 0 & 0 & 0 & 0 & 2 & 0 & 0.5 & 0 & 0 & 0 & 2.5 \\
\hline Fusarium & 6 & 3.5 & 2 & 3 & 2.5 & 4.5 & 2.5 & 2.5 & 3 & 3.5 & 3.5 & 5 & 41.5 \\
\hline Epicoccum & 0 & 2 & 2.5 & 0 & 0 & 1.5 & 1.5 & 1.5 & 0 & 0 & 3 & 0 & 12 \\
\hline Hendersonia sp. & 13 & 6 & 11 & 8 & 7.5 & 7 & 7 & 5 & 10 & 8.5 & 4 & 8.5 & 95.5 \\
\hline Ascochyta sp. & 5.5 & 7 & 13 & 8 & 16 & 5.5 & 8 & 9.5 & 5.5 & 8.5 & 6 & 5 & 97.5 \\
\hline Diplodia sp. & 8 & 18.5 & 12.5 & 9.5 & 8.5 & 11 & 6.5 & 11.5 & 7.5 & 6.5 & 6.5 & 6.5 & 113 \\
\hline Sphaeropsis sp. & 0 & 0 & 1.5 & 0 & 0 & 0 & 0 & 0 & 1 & 0 & 0 & 0 & 2.5 \\
\hline Unidentified spores & 0 & 2 & 1 & 1 & 0 & 0 & 1 & 0 & 0 & 0 & 0 & 0.5 & 5.5 \\
\hline Total spores & 177.5 & 251.5 & 338.5 & 373 & 249.5 & 229 & 163.5 & 223 & 226 & 247.5 & 228.5 & 222 & 2929.5 \\
\hline
\end{tabular}

Table 5. The identified airborne fungi genera or species and colony forming count at 10 meter during 12 months.

\begin{tabular}{|c|c|c|c|c|c|c|c|c|c|c|c|c|c|}
\hline or species & Jan & Feb & Mar & Apr & May & Jun & Jul & Aug & Sep & Oct & Nov & Dec & $\begin{array}{l}\text { Total } \\
\text { colony }\end{array}$ \\
\hline Mucor & 0 & 157 & 157 & 0 & 0 & 0 & 0 & 0 & 0 & 0 & 0 & 0 & 314 \\
\hline Rhizopus & 0 & 0 & 0 & 0 & 157 & 0 & 0 & 0 & 0 & 0 & 0 & 0 & 157 \\
\hline Chaetomium & 0 & 0 & 0 & 0 & 0 & 157 & 0 & 0 & 157 & 0 & 0 & 0 & 314 \\
\hline Saccharomyces & 157 & 472 & 786 & 944 & 472 & 629 & 157 & 472 & 315 & 315 & 472 & 472 & 5663 \\
\hline Penicillium & 157 & 944 & 1258 & 2674 & 472 & 786 & 315 & 315 & 2202 & 1887 & 1415 & 472 & 12897 \\
\hline Aspergillus & 315 & 472 & 315 & 2045 & 315 & 1573 & 315 & 315 & 1101 & 1887 & 472 & 157 & 9282 \\
\hline Cladosporium & 786 & 1258 & 472 & 315 & 472 & 315 & 315 & 472 & 629 & 315 & 315 & 629 & 6293 \\
\hline Trichothecium & 0 & 157 & 0 & 0 & 0 & 0 & 0 & 157 & 0 & 0 & 0 & 0 & 314 \\
\hline Trichoderma & 0 & 315 & 0 & 0 & 315 & 0 & 0 & 0 & 0 & 0 & 0 & 0 & 630 \\
\hline Botrytis & 0 & 0 & 157 & 0 & 0 & 0 & 0 & 0 & 0 & 157 & 0 & 0 & 314 \\
\hline Geotrichum & 0 & 0 & 0 & 0 & 0 & 0 & 0 & 157 & 315 & 0 & 0 & 0 & 472 \\
\hline Alternaria & 0 & 0 & 629 & 157 & 157 & 0 & 0 & 315 & 315 & 157 & 157 & 0 & 1887 \\
\hline Curvularia & 0 & 0 & 315 & 0 & 157 & 0 & 0 & 0 & 157 & 629 & 0 & 157 & 1415 \\
\hline Helminthosporium & 0 & 157 & 157 & 0 & 0 & 315 & 0 & 157 & 0 & 0 & 157 & 0 & 943 \\
\hline Nigrospora & 0 & 0 & 0 & 0 & 0 & 0 & 0 & 0 & 157 & 0 & 0 & 0 & 157 \\
\hline Stachybotrys & 0 & 0 & 157 & 0 & 0 & 0 & 0 & 0 & 0 & 157 & 0 & 0 & 314 \\
\hline Fusarium & 0 & 157 & 0 & 157 & 0 & 0 & 0 & 0 & 157 & 0 & 0 & 0 & 471 \\
\hline Phoma sp. & 0 & 0 & 157 & 0 & 0 & 0 & 0 & 157 & 0 & 0 & 0 & 0 & 314 \\
\hline $\begin{array}{l}\text { Non-sporulating } \\
\text { fungi }\end{array}$ & 0 & 629 & 629 & 2045 & 629 & 1101 & 157 & 944 & 1887 & 1415 & 786 & 786 & 11008 \\
\hline Unidentified fungi & 157 & 0 & 0 & 0 & 0 & 0 & 0 & 0 & 0 & 0 & 157 & 0 & 314 \\
\hline Total colony & 1572 & 4718 & 5189 & 8337 & 3146 & 4876 & 1259 & 3461 & 7392 & 6919 & 3931 & 2673 & 53473 \\
\hline
\end{tabular}


Table 6. The identified airborne fungi genera or species and colony forming count at 30 meter during 12 months.

\begin{tabular}{|c|c|c|c|c|c|c|c|c|c|c|c|c|c|}
\hline or species & Jan & Feb & Mar & Apr & May & Jun & Jul & Aug & Sep & Oct & Nov & Dec & $\begin{array}{c}\text { Total } \\
\text { colony }\end{array}$ \\
\hline Mucor & 0 & 0 & 0 & 0 & 0 & 157 & 0 & 0 & 0 & 0 & 0 & 0 & 157 \\
\hline Rhizopus & 0 & 0 & 0 & 0 & 0 & 157 & 0 & 0 & 0 & 0 & 0 & 0 & 157 \\
\hline Chaetomium & 0 & 157 & 0 & 0 & 0 & 0 & 0 & 0 & 0 & 0 & 0 & 0 & 157 \\
\hline Saccharomyces & 157 & 629 & 472 & 1101 & 629 & 315 & 629 & 315 & 315 & 315 & 472 & 315 & 5664 \\
\hline Penicillium & 315 & 1101 & 1573 & 2045 & 629 & 629 & 472 & 629 & 2045 & 2359 & 1101 & 472 & 13370 \\
\hline Aspergillus & 472 & 472 & 1573 & 1730 & 315 & 315 & 629 & 472 & 629 & 629 & 315 & 315 & 7866 \\
\hline Cladosporium & 472 & 472 & 315 & 472 & 315 & 1101 & 0 & 629 & 315 & 315 & 157 & 157 & 4720 \\
\hline Trichothecium & 0 & 0 & 157 & 0 & 0 & 0 & 0 & 0 & 0 & 315 & 0 & 0 & 472 \\
\hline Trichoderma & 0 & 0 & 0 & 0 & 0 & 157 & 0 & 0 & 0 & 0 & 0 & 0 & 157 \\
\hline Botrytis & 0 & 157 & 0 & 0 & 0 & 0 & 0 & 0 & 0 & 0 & 0 & 0 & 157 \\
\hline Geotrichum & 0 & 0 & 0 & 0 & 0 & 0 & 0 & 0 & 0 & 0 & 0 & 0 & 0 \\
\hline Alternaria & 0 & 315 & 0 & 157 & 0 & 472 & 0 & 157 & 944 & 315 & 157 & 157 & 2674 \\
\hline Curvularia & 0 & 157 & 315 & 0 & 0 & 157 & 0 & 629 & 315 & 786 & 472 & 472 & 3303 \\
\hline Helminthosporium & 0 & 157 & 0 & 0 & 0 & 0 & 157 & 0 & 0 & 157 & 0 & 0 & 471 \\
\hline Nigrospora & 0 & 0 & 0 & 0 & 0 & 0 & 0 & 0 & 315 & 0 & 0 & 0 & 315 \\
\hline Stachybotrys & 0 & 157 & 0 & 0 & 0 & 0 & 0 & 0 & 0 & 0 & 0 & 0 & 157 \\
\hline Fusarium & 0 & 0 & 0 & 0 & 0 & 157 & 0 & 0 & 0 & 0 & 0 & 0 & 157 \\
\hline Phoma sp. & 0 & 0 & 0 & 0 & 0 & 0 & 0 & 0 & 157 & 157 & 0 & 0 & 314 \\
\hline Non-sporulating fungi & 472 & 315 & 1258 & 2516 & 315 & 629 & 0 & 786 & 1415 & 1730 & 472 & 629 & 10537 \\
\hline Unidentified fungi & 0 & 0 & 0 & 0 & 157 & 0 & 0 & 0 & 0 & 0 & 0 & 0 & 157 \\
\hline Total colony & 1888 & 4089 & 5663 & 8021 & 2360 & 4246 & 1887 & 3617 & 6450 & 7078 & 3146 & 2517 & 50962 \\
\hline
\end{tabular}

Table 7. The identified airborne fungi genera or species and colony forming count at 70 meter during 12 months.

\begin{tabular}{|c|c|c|c|c|c|c|c|c|c|c|c|c|c|}
\hline$\underbrace{\text { Genera month }}_{\text {or species }}$ & Jan & Feb & Mar & Apr & May & Jun & Jul & Aug & Sep & Oct & Nov & Dec & $\begin{array}{c}\text { Total } \\
\text { colony }\end{array}$ \\
\hline Mucor & 0 & 0 & 0 & 0 & 0 & 0 & 0 & 0 & 0 & 0 & 0 & 0 & 0 \\
\hline Rhizopus & 0 & 157 & 0 & 0 & 0 & 0 & 0 & 0 & 0 & 0 & 0 & 0 & 157 \\
\hline Chaetomium & 0 & 157 & 0 & 0 & 0 & 0 & 0 & 0 & 0 & 157 & 0 & 0 & 314 \\
\hline Saccharomyces & 472 & 1258 & 786 & 786 & 315 & 629 & 315 & 786 & 786 & 944 & 472 & 629 & 8178 \\
\hline Penicillium & 472 & 315 & 1730 & 2045 & 944 & 944 & 472 & 472 & 629 & 1415 & 944 & 472 & 10854 \\
\hline Aspergillus & 472 & 315 & 472 & 1258 & 315 & 786 & 472 & 472 & 786 & 944 & 629 & 0 & 6921 \\
\hline Cladosporium & 786 & 472 & 1101 & 629 & 472 & 629 & 315 & 472 & 786 & 472 & 629 & 472 & 7235 \\
\hline Trichothecium & 0 & 0 & 0 & 0 & 0 & 0 & 0 & 0 & 157 & 157 & 0 & 0 & 314 \\
\hline Trichoderma & 0 & 0 & 0 & 157 & 0 & 0 & 0 & 0 & 0 & 0 & 157 & 0 & 314 \\
\hline Botrytis & 0 & 0 & 0 & 0 & 0 & 157 & 0 & 0 & 0 & 0 & 157 & 0 & 314 \\
\hline Alternaria & 0 & 472 & 472 & 472 & 157 & 315 & 0 & 472 & 315 & 157 & 157 & 315 & 3304 \\
\hline Curvularia & 0 & 0 & 0 & 157 & 157 & 472 & 0 & 157 & 786 & 944 & 0 & 315 & 2988 \\
\hline Helminthosporium & 0 & 0 & 0 & 157 & 157 & 0 & 0 & 0 & 157 & 0 & 0 & 0 & 471 \\
\hline Nigrospora & 0 & 0 & 0 & 0 & 0 & 0 & 0 & 0 & 315 & 0 & 0 & 0 & 315 \\
\hline Stachybotrys & 0 & 0 & 0 & 0 & 0 & 157 & 0 & 0 & 0 & 0 & 157 & 0 & 314 \\
\hline Fusarium & 0 & 157 & 315 & 0 & 0 & 0 & 0 & 0 & 0 & 0 & 0 & 0 & 472 \\
\hline Phoma sp. & 0 & 0 & 0 & 0 & 0 & 0 & 0 & 0 & 0 & 0 & 157 & 157 & 314 \\
\hline Non-sporulating fungi & 0 & 315 & 629 & 1573 & 786 & 786 & 472 & 315 & 315 & 629 & 315 & 472 & 6607 \\
\hline Unidentified fungi & 0 & 0 & 0 & 0 & 0 & 0 & 0 & 0 & 0 & 157 & 0 & 0 & 157 \\
\hline Total colony & 2202 & 3618 & 5505 & 7234 & 3303 & 4875 & 2046 & 3146 & 5032 & 5976 & 3774 & 2832 & 49543 \\
\hline
\end{tabular}




\subsection{Effect of Seasonal Variations on the Total Airborne Fungi in One Year}

The airborne fungal spores were present in the air around Shenzhen University throughout the year. The distribution curve of total airborne fugal spores peaked in April and October, and dropped to the lowest values in January, July and December (Figure 1). The distribution curve of the total colony forming unit spiked in April, October and September, and fell to the lowest values in January, July and December (Figure 2).

\subsection{The Effect of Seasonal Variations on Airborn Fungi at Three Different Altitudes}

At 10, 30 and 70 meters height, increased levels of total airborne fugal spores was observed during April and October, while lower levels were observed during January, July and December (Figure 3). The concentration of fungal spores decreased with respect to the increase in the altitude. The number of spores at 70 meters was lower compared to the levels observed at 30 and 10 meters during September and October.

The distribution curve of fungal colony at 10, 30 and 70 meters height indicated a sharp increase during four months from January to April. The distribution curve of the fungal colonies at 10 and 70 meters reached to its lowest point during July, January, December, and at 30 meter height similar levels were observed in July, January and May (Figure 4).

\subsection{The Relationship between the Airborne Fungal and Meteorological Factors}

The monthly meteorological measurements: average temperature, average relative humidity, average atmospheric pressure, average wind speed, rainfall and solar radiation had no observable relationship between the total spore count at 10 and 30 meters height. At 70 meters height, the total spore count was negatively correlated with solar radiation $(\mathrm{Y}=342.191-0.718 \mathrm{X} 6, \mathrm{r}=0.602, \mathrm{p}<0.05)$.

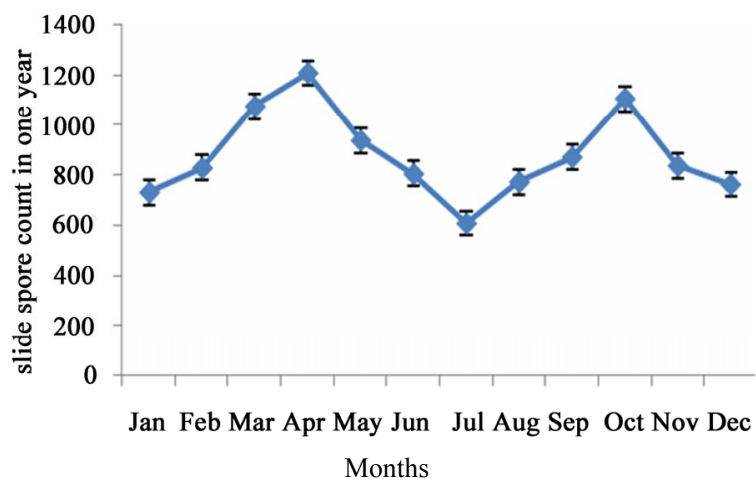

Figure 1. The distribution of slide spore count during a 12 month period.

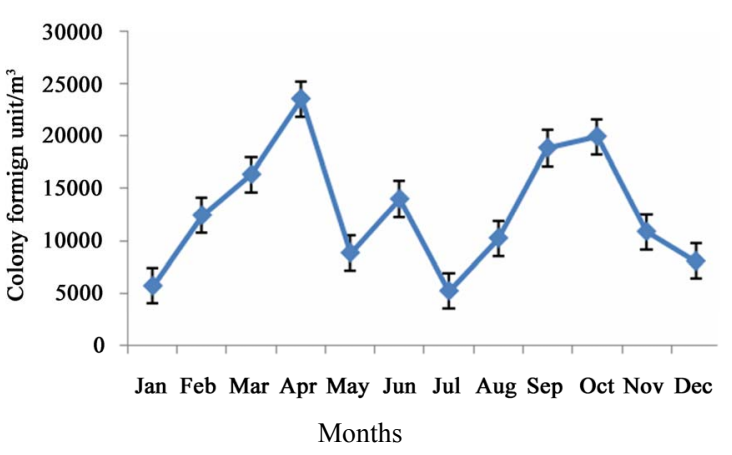

Figure 2. The distribution of colony forming count during a 12 month period.

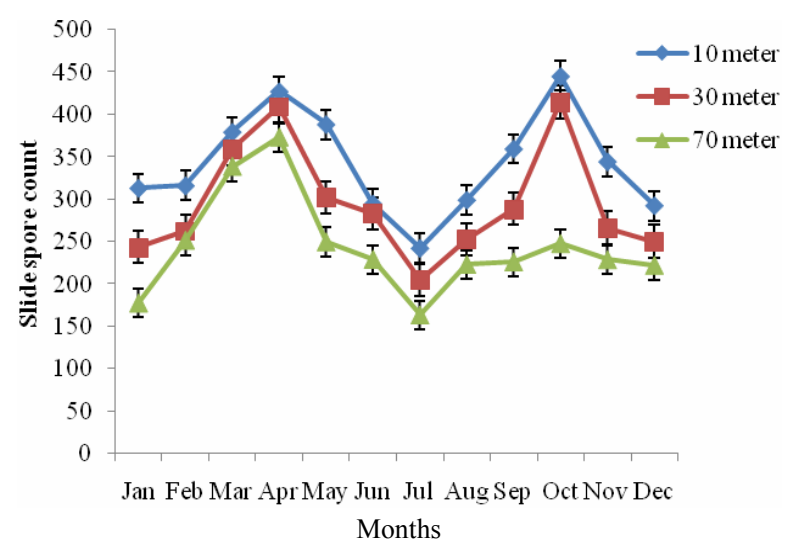

Figure 3. The distribution of slide spore count at three altitudes during a 12 month period.

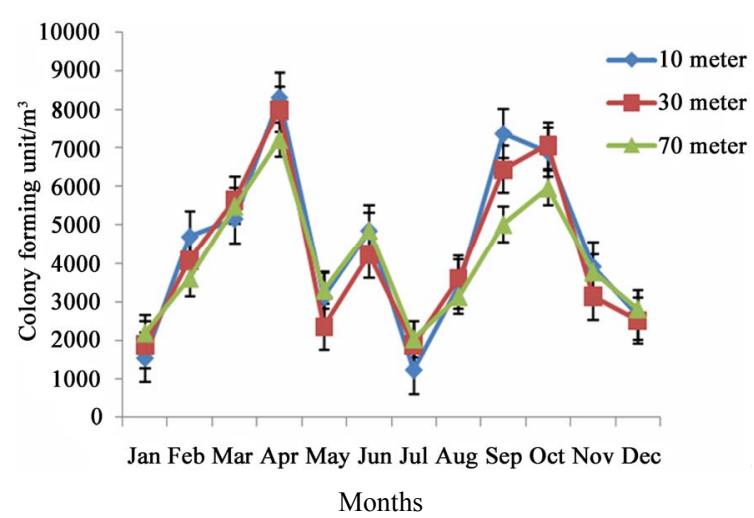

Figure 4. The distribution of colony forming count at three altitudes during a 12 month period.

The total monthly spore count of Cladosporium, Ustilago, Alremaria had no relationship between the meteorological factors. The spore counts of Holminthsporium was negatively correlated with solar radiation $(\mathrm{Y}=$ $156.026-0.409 \mathrm{X} 6, \mathrm{r}=0.602, \mathrm{p}<0.05)$. The spore count of Uredinales was negatively correlated with average wind speed and rainfall $(\mathrm{Y}=176.894-35.314 \mathrm{X} 4$ $-0.099 \mathrm{X} 5, \mathrm{r}=0.805, \mathrm{p}<0.05)$. 


\section{DISCUSSION}

\subsection{Comparison of Airborne Fungi in Shenzhen University and Other Regions}

The most common fungi documented in many Chinese districts were Alremari, Cladosporium, Ustilago, Uredinales, Aspergillus, Penicillium, Holminthsporium, Fusarium and Saccharomyces [16-25]. Similar observations were documented in many other countries as well [7-9,12,14,28,29]. The top five genera of fungi spores in our study were Cladosporium (16.45\%), Ustilago (13.04\%), Alremaria (11.41\%), Holminthsporium $(11.37 \%)$ and Uredinales $(9.54 \%)$. While the top five genera of fungi spore in Guangzhou were Alremaria (27.49\%), Ustilago (17.26\%), Uredinales (6.95\%), Holminthsporium (6.09\%) and Fusarium (4.99\%). Cladosporium only took up $0.77 \%$ in Guangzhou [16]. The top five genera of fungal colony in our study were Penicillium (24.11\%), Aspergillus (15.63\%), Saccharomyces (12.67\%), Cladosporium (11.85\%) and Alremaria $(5.11 \%)$. The top five genera of fungi colony in Guangzhou were Cladosporium (21.60\%), Penicillium (19.71\%), Alremaria (5.54\%), Rhizopus (5.05\%) and Aspergillus $(4.43 \%)$. The non-sporulating fungi in our study area and in Guangzhou were $18.28 \%$ and $30.79 \%$, respectively [16]. This indicates that the fungal spore count differs according to time and place.

The concentration of fungal spores changed with seasonal variation in many districts in China during April to October [16-25]. The concentration of fungal spores in Guangzhou was high during April, September and October, and the lower during January, July and December [16]. Similar results were observed in our study.

\subsection{Comparison of Airborne Fungal Spores at Different Altitudes}

Our results showed that the total spore count decreased with the increase in height. However, the total genera of fungi did not decrease with the height. The fungi with larger spore sizes, such as Holminthsporium, Alremaria, Ustilago and Curvularia were concentrated at 10 meters height. While the fungi with smaller spore sizes such as Cladosporium was concentrated at 70 meters. The count of Aspergillus didn't show much difference at 30 or 70 meters height. Chakraborty (2001) [13] reported that the smaller spores were dominant at greater heights and larger spores and conidia were more prevalent at lower levels. Furthermore, the distribution of fungal spores at different altitude was influenced by their shapes [30].

\subsection{The Relationship between the Airborne Fungi and Meteorological Factors}

The distribution of airborne fungi spores can be affected by various factors including meteorological factors. The effect of meteorological factors on the count of airborne fungal spores varied from one fungal taxon to another. Most airborne fungi have a strong relationship with temperature; however, Aspergillus/Penicillium hyphal fragments were positively correlated with wind speed. In comparison with other airborne fungi, Leptosphaeria and unidentified Ascomycetes were more closely correlated with rain and relative humidity during the growing season [31]. Alternaria and Cladosporium are positively correlated with temperature and duration of sunlight. However, Ustilago indicated a positive correlation with relative humidity and negative correlation with wind speed [32]. Alternaria and Cladosporium showed a positive association with temperature, duration of sunlight and accumulated rainfall, but negatively correlated with daily rainfall [33].

In our results, the meteorological factors had no observable relationship between the total monthly spore count and altitude. At 70 meters, the total spore count was negatively correlated with solar radiation. The total monthly spore count of Cladosporium, Ustilago, Alremaria had no observable relationship between the meteorological factors. The spore count of Holminthsporium was negatively correlated with solar radiation, while the spore count of Uredinales was negatively correlated with average wind speed and rainfall. In this study, the temperature, relative humidity, atmospheric pressure did not affect the total spore concentrations. It may be due to the subtropical climatic location of Shenzhen. The meteorological data showed that the temperature, relative humidity, atmospheric pressure did not change drastically in Shenzhen during the entire year. Therefore, seasonal variations did not affect the distibution of the fungal spores significantly.

\section{CONCLUSIONS}

There were 27 genera or species of fungus spores and 18 genera of fungi colonies identified in a given year. The airborne fungal spores were present in the atmosphere of Shenzhen University all year round. The peaks of airborne spores appeared during April and October, while the lowest numbers were observed during January, July and December. The highest volumes of fungi colonies were observed during April, October and September, while the lowest numbers were detected during in January, July and December or May. The meteorological factors had no relationship between the total monthly spore count at 10 and 30 meter height. At 70 meter, the total spores count was negatively correlated with solar radiation. Most of fungi spores decreased along with the increase of altitudes.

\section{ACKNOWLEDGEMENTS}

This study was financially supported by the natural science foundation of Guangdong Province (No. 04300891). The authors would like to gratefully acknowledge Guo- qiang Xiong and Yan Ying in center for 
disease control of Nanchang who helped with the indentification of the fungi. The authors would also thank Shenzhen meteorlogical administration for providing the meteorlogical data.

\section{REFERENCES}

[1] Horner, W.E., Helbling, A., Salvaggio, J.E. and Lehrer, S.B. (1995) Fungal allergens. Clinical Microbiology Reviews, 8(2), 161-179.

[2] Niedoszytko, M., Chełmińska, M. and Chełmiński, K. (2002) Fungal allergy--part II. Polski Merkuriusz Lekarski, 12(70), 314-317.

[3] Semik-Orzech, A., Barczyk, A. and Pierzchała, W. (2008) The influence of sensitivity to fungal allergens on the development and course of allergic diseases of the respiratory tract. Pneumonologia I Alergologia Polska, 76(1), 29-36.

[4] Kurup, V.P., Shen, H.D. and Banerjee, B. (2000) Respiratory fungal allergy. Microbes and Infection, 2(9), 1101-1110.

[5] Kakde, U.B., Kakde, H.U. and Saoji, A.A. (2001) Seasonal variation of fungal propagules in a fruit market environment, Nagpur (India). Aerobiologia, 17, 177-182.

[6] Katial, R.K., Zhang, Y., Jones, R.H. and Dyer, P.D. (1997) Atmospheric mold spore counts in relation to meteorological parameters. International Journal of Biometeorology, 41(1), 17-22.

[7] Oliveira, M., Ribeiro, H. and Abreu, I. (2005) Annual variation of fungal spores in atmosphere of Porto: 2003. Annals of Agricultural and Environmental Medicine, 12(2), 309-315.

[8] Ogunlana, E.O. (1975) Fungal air spora at Ibadan, Nigeria. Journal of Applied Microbiology, 29(4), 458-463.

[9] Moustafa, A.F. and Kamel, S.M. (1976) A study of fungal spore poupulations in the atmosphere of Kuwait. Mycopathologia, 59(1), 29-35.

[10] Takahashi, T. (1997) Airborne fungal colony-forming units in outdoor and indoor environments in Yokohama, Japan. Mycopathologia, 139, 23-33.

[11] Marshall, W.A. (1997) Seasonality in antarctic airborne fungal spores. Applied and Environmental Microbiology, 63(6), 2240-2245.

[12] Al-Suwaine, A.S., Bahkali, A.H. and Hasnain, S.M. (1999) Seasonal incidence of airborne fungal allergens in Riyadh, Saudi Arabia. Mycopathologia, 145(1), 15-22.

[13] Chakraborty, P., Gupta-Bhattacharya, S., Chowdhury, I., Majumdar, M.R. and Chanda, S. (2001) Differences in concentrations of allergenic pollens and spores at different heights on an agricultural farm in West Bengal, India. Annals of Agricultural and Environmental Medicine, 8(2), 123-130.

[14] Mezzari, A., Perin, C., Santos Júnior, S.A., Bernd, L.A. and Gesu, G.D. (2003) Airborne fungi and sensitization in atopic individuals in Porto Alegre, RS, Brazil. Revista da Associação Médica Brasileira, 49(3), 270-273.

[15] Segvić Klarić, M. and Pepeljnjak, S. (2006) A year-round aeromycological study in Zagreb area, Croatia. Annals of Agricultural and Environmental Medicine, 13(1), 55-64.

[16] Zhan, Q.S., Wang, S.H., Li, M.L. and Zhang, W.Y. (1994) Study on quantity and types of allergic fungi in air. Modern Preventive Medicine, 21(2), 67-68. (in Chinese)

[17] Jiang, X.W., Wu, W.J., Yang, C.Y. and Jiang, X.C. (1994)
A primanry investigation on airborne allergenic fungi in the eastern district of Changsha. Bulletin of Hunan Medical University, 19(4), 326-328. (in Chinese)

[18] Li, Y.F. and Sun, X.Z. (1994) Investigation of airborne fungi in Xian distict. Journal of Xian Medical University, 15(4), 371-382. (in Chinese)

[19] Fang, R.Q., Xie, S.Q. and Zhan, H.M. (1997) Investigation report of airborne fungus across Kunming city proper. Academic Journal of Kunming Medical College, 18(3), 30-33. (in Chinese)

[20] Wang, M.M. and Liu, T.M. (1997) Prodominant fungi present in the air of a hospital in Nanjing and their seasonal variation. Journal of Nanjing Railway Medical College, 16(4), 256-258. (in Chinese)

[21] Zhang, Z.Z., Li, Y., Long, F.Q. and Mo, X. (1999) Investigation of airborne fungi in Nanning Guangxi Province. Guangxi Medical Journal, 21(6), 1121-1124. (in Chinese)

[22] Ning, Y.L., Chang, H.Z. and Zhang, N. (2001) Investigation report of airborne fungus in Baoding City. Journal of Hebei Medical College Continuting Education, 18(3), 40-41. (in Chinese)

[23] Su, H., Lu, X., Chen, W.Y., Zhang, B.B. and Li, Y.J. (2001) Investigation on the atmosphere propagating sensitzing Fungi in Wuhan. Chinese Journal of Microbiology and Immunology, 21(Supplement), 42-43. (in Chinese)

[24] Jin, Z.C., Yin, K.H. and Chen, X.X. (2001) Study of the relationship between airborne fung and asthma in Zhenjiang city. Practical Preventive Medicine, 8(3), 170-172. (in Chinese)

[25] Huang, J.J., Hong, S.L., Zhou, W. and Yang, Y.C. (2002) Air-borne molds in Chongqing city. Chongqing Medicine, 31(8), 701-703. (in Chinese)

[26] Ye, S.T., Qiao, B.S. and Lu, Y.J. (1992) China allergic aeromycology. People's Medical Publishing House, Beijing. (in Chinese)

[27] Zhou, S.N., Chen, W.T., Burnett, J. and Deng, B.L. (1997) A study of microorganisms in air-conditioned indoor environment. Acta Scientiae Circumstantiae, 17(4), 498-501. (in Chinese)

[28] Hedayati, M.T., Mayahi, S., Aghili, R. and Goharimoghadam, K. (2005) Airborne fungi in indoor and outdoor of asthmatic patients' home, living in the city of sari. Iranian Journal of Allergy, Asthma and Immunology, 4(4), 189-91.

[29] Ana, S.G., Torres-Rodríguez, J.M., Ramírez, E.A., García, S.M. and Belmonte-Soler, J. (2006) Seasonal distribution of Alternaria, Aspergillus, Cladosporium and Penicillium species isolated in homes of fungal allergic patients. Journal Investigational Allergology and Clinical Immunology, 16(6), 357-363.

[30] Lacey, J. (1997). Fungi and actinomycemycetes as allergens. In: Kay, A.B. Ed., Allergy and Allergic Diseases, Black Science, London K, 858-883.

[31] Li, D.W. and Kendrick, B. (1995) A year-round study on functional relationships of airborne fungi with meteorological factors. International Journal of Biometeorology, 39(2), 74-80.

[32] Sabariego, S., Guardia, C.D. and Alba, F. (2000) The effect of meteorological factors on the daily variation of airborne fungal spores in Granada (southern Spain). International Journal of Biometeorology, 44(1), 1-5. 
[33] Sabariego, S., Guardia, C.D. and Alba, F. (2004) Aerobiological study of Alternaria and Cladosporium conidia in the atmosphere of Almeria (SE Spain). Revista Iberoamericana de Micología, 21(3), 121-127. 